\title{
Synthesis, characterization and anticancer properties of organometallic Schiff base platinum complexes
}

\begin{tabular}{|r|l|}
\hline Journal: & Canadian Journal of Chemistry \\
\hline Manuscript ID: & cjc-2015-0157.R1 \\
\hline Manuscript Type: & Article \\
\hline Date Submitted by the Author: & O2-May-2015 \\
\hline Complete List of Authors: & $\begin{array}{l}\text { Jean, Stéphanie; Université de Moncton, Département de chimie et } \\
\text { biochimie } \\
\text { Cormier, Kevin; Université de Moncton, Département de chimie et } \\
\text { biochimie } \\
\text { Patterson, Alyssa; Mount Allison University, Chemistry } \\
\text { Vogels, Christopher; Mount Allison University, Chemistry } \\
\text { Decken, Andreas; Department of Chemistry } \\
\text { Robichaud, Gilles; Université de Moncton, Département de chimie et } \\
\text { biochimie } \\
\text { Turcotte, Sandra; Université de Moncton, Département de chimie et } \\
\text { biochimie } \\
\text { Westcott, Stephen; Mount Allison University }\end{array}$ \\
\hline Keyword: & \begin{tabular}{l} 
Anticancer, Cytotoxicity, Platinum, Organometallic, Schiff-base \\
\hline
\end{tabular} \\
\hline
\end{tabular}


Synthesis, characterization and anticancer properties of organometallic Schiff base platinum complexes

Stéphanie Jean, $\uparrow$ Kevin Cormier, $\dagger$ Alyssa E. Patterson, $\uparrow$ Christopher M. Vogels, Andreas

Decken, Gilles A. Robichaud*, Sandra Turcotte*, Stephen A. Westcott*

S. Jean, K. Cormier, G.A. Robichaud, and S. Turcotte. Département de chimie et biochimie, Université de Moncton, Campus de Moncton, Moncton, NB E1A 3E9, Canada and the Atlantic Cancer Research Institute, Moncton, NB E1C 8X3, Canada.

A.E. Patterson, C.M. Vogels and S.A. Westcott. Department of Chemistry and Biochemistry, Mount Allison University, Sackville, NB E4L 1G8, Canada.

A. Decken. Department of Chemistry, University of New Brunswick, Fredericton, NB E3B 5A3, Canada

Corresponding author: Stephen A. Westcott (e-mail: swestcott@mta.ca).

Corresponding authors for anticancer studies: Gilles A. Robichaud (e-mail: gilles.robichaud@umoncton.ca) and Sandra Turcotte (sandra.turcotte@umoncton.ca).

Corresponding author for X-ray studies: Andreas Decken (e-mail: adecken@unb.ca).

$\dagger$ These authors contributed equally to this work. 


\section{Abstract:}

A small family of organometallic platinum complexes containing a chloride, ciscyclooctene, and a Schiff base ligand have been prepared and characterized fully. Three aliphatic amines and four aromatic amines were chosen as representative examples. All complexes were stable in air except for $\mathbf{7}$, derived from the pinacol-protected 4aminophenylboronate ester 4- $\mathrm{H}_{2} \mathrm{NC}_{6} \mathrm{H}_{4}$ Bpin (pin = 1,2- $\mathrm{O}_{2} \mathrm{C}_{2} \mathrm{Me}_{4}$ ), which decomposed via B-C bond cleavage. Both complex 4 (derived from aniline) and $\mathbf{7}$ were further characterized by single crystal X-ray diffraction studies and confirmed the square planar nature of the platinum center whereby the chloride ligand lies trans to the deprotonated hydroxyl group of the Schiff base ligand. The imine functionality is trans to the organic cyclooctene group. Complex 3, which contained the longest aliphatic chain studied (an octyl group), was the most promising for inducing apoptosis in the malignant MB231 breast cancer cell line. Conversely, complexes 4-6, which contained aromatic groups, were the most active against Renal Cell Carcinoma (RCC) cell lines.

Keywords: Anticancer; Cytotoxicity; Platinum; Organometallic; Schiff base

\section{Graphical Abstract:}

Seven new organometallic (salicylaldiminato)platinum(II) complexes have been prepared, and characterized fully, whereupon six of these new compounds were examined for their in vitro cytotoxic properties against breast cancer cell line MB231 and Renal Cell Carcinoma (RCC) cell lines.

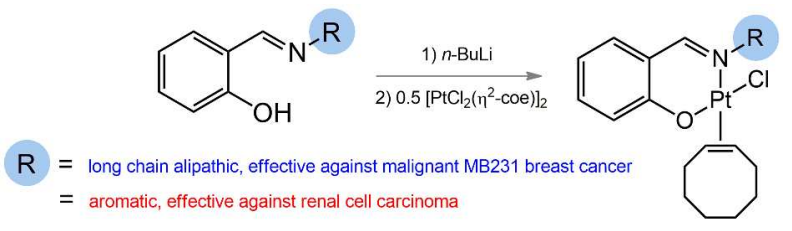




\section{Introduction}

Cisplatin, or cis-diamminedichloridoplatinum(II) (Fig. 1), is a remarkable small molecule that has made a large impact in the world of medicinal inorganic chemistry. Indeed, cisplatin has been used in the treatment of metastatic ovarian and testicular cancers for over thirty years, working primarily through a mechanism involving cross-linking DNA and inhibiting transcription.1,2 Cisplatin is believed to enter the cell where it is purported to undergo aquation with loss of one or both of the chloride ligands. The resulting cationic Lewis acidic platinum atom can then readily bind to a number of biological ligands, including the purine bases of DNA. Unfortunately, this form of chemotherapy is only useful for a limited number of cancers and suffers from serious side effects arising from the poor solubility of cisplatin in physiological media. A myriad of second generation platinum compounds have been prepared as potential anticancer drugs over the last forty years but most are based on altering either the inert amine groups or varying the leaving groups. While some improvements have been made in this area, including the use of $\mathrm{Pt}(\mathrm{IV})$ complexes for improved drug delivery, ${ }^{3}$ similar problems that are associated with cisplatin are observed with these next-generation anticancer derivatives. New strategies are therefore required to bring inorganic compounds to the next level of chemotherapy.4,5

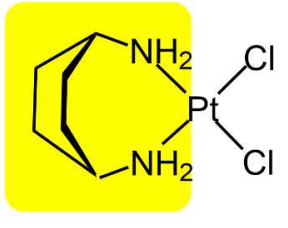

Cis-[PtCl $2(1,4-D A C H)]$ -variation of inert ammine groups

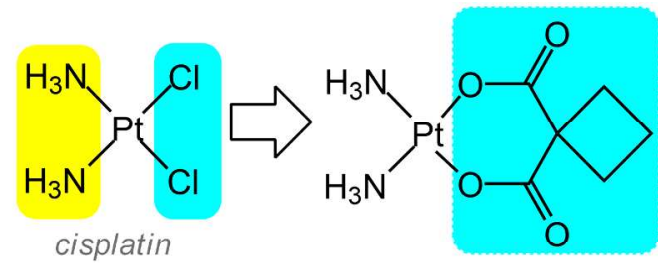

carboplatin

-variation of leaving groups

Fig. 1. Cisplatin, and its derivatives, cis- $\left[\mathrm{PtCl}_{2}(1,4-\mathrm{DACH})\right]$ (DACH = diaminocyclohexane) and carboplatin. 
A recent paper by Gust and co-workers demonstrated that salicylaldimine complexes of platinum also displayed significant in vitro antitumour activity against MCF-7 human breast cancer cells. 6 Variation of the salicylaldimine groups greatly altered the growth inhibitory effects for these compounds and improved activities were observed with electron withdrawing groups. Encouraged by these results, and our own interest in developing non-traditional platinum anticancer complexes, we have also reported that bis(salicylaldiminato)platinum(II) complexes had appreciable cytotoxic activity against three human glioma cell lines. ${ }^{7}$ Unfortunately, these complexes suffered from poor solubility, limiting any additional studies. To circumvent this issue, we designed a few organic-soluble cis-chlorido(cis-cyclooctene)(salicylaldiminato)platinum(II) complexes that showed considerable promise against three human glioma cell lines. The use of organometallic platinum complexes as potential cytotoxic agents is an area of growing interest, providing an alternative to traditional coordination complexes based on the structural motif of cisplatin. ${ }^{8-10}$ In this present study, we have expanded upon our initial findings in this area and prepared new organometallic platinum complexes to investigate if these types of compounds are also cytotoxic against other types of cancer, such as breast cancer and Renal Cell Carcinoma (RCC). RCC is the major type of kidney cancer (85\% of cases) and metastatic RCC are resistant to standard chemotherapies including cisplatin.

\section{Experimental}

\section{General methods and materials}

Reagents and solvents used were obtained from Aldrich Chemicals. $\left[\mathrm{PtCl}_{2}\left(\eta^{2}-\mathrm{coe}\right)\right]_{2}$ (coe $=$ cis-cyclooctene) ${ }^{11}$ and Schiff bases ${ }^{12}$ were synthesized according to literature procedures. NMR spectra were recorded on a JEOL JNM-GSX400 FT NMR (1H: 400 
$\mathrm{MHz}$; ${ }^{11 \mathrm{~B}:} 128 \mathrm{MHz}$; $\left.{ }^{13} \mathrm{C}: 100 \mathrm{MHz} ;{ }^{19} \mathrm{~F}: 376 \mathrm{MHz}\right)$ spectrometer. Chemical shifts $(\delta)$ are reported in ppm [relative to residual solvent peaks $\left({ }^{1} \mathrm{H}\right.$ and $\left.{ }^{13} \mathrm{C}\right)$ or external $\mathrm{BF}_{3} \cdot \mathrm{OEt}_{2}\left({ }^{11} \mathrm{~B}\right)$ and $\left.\mathrm{CF}_{3} \mathrm{CO}_{2} \mathrm{H}\left({ }^{19} \mathrm{~F}\right)\right]$. Multiplicities are reported as singlet $(\mathrm{s})$, doublet $(\mathrm{d})$, triplet $(\mathrm{t})$, multiplet $(\mathrm{m})$, broad (br) and overlapping (ov) with coupling constants $(J)$ reported in hertz. FT-IR spectra were obtained with a Thermo Fisher Scientific Nicolet iS5 FT-IR spectrometer in ATR mode and are reported in $\mathrm{cm}^{-1}$. Melting points were measured uncorrected with a Stuart SMP30 apparatus. Elemental analyses for C, H, and N were carried out at Guelph Chemical Laboratories (Guelph, ON) and Laboratoire d'Analyse Élémentaire de l'Université de Montréal (Montréal, QC). All reactions were performed under an atmosphere of dinitrogen. Platinum compounds were isolated under ambient conditions except for $\mathbf{7}$ which is unstable in air.

\section{General synthesis of mono Schiff base platinum complexes}

Under an atmosphere of dinitrogen, a hexane solution of $n$-BuLi (1.0 mL of a $1.6 \mathrm{M}$ solution, $1.6 \mathrm{mmol})$ was added to a toluene $(5 \mathrm{~mL})$ solution of the appropriate Schiff base (1.60 mmol). Upon stirring the reaction mixture for $3 \mathrm{~h}$ at $\mathrm{RT}$, a toluene $(20 \mathrm{~mL})$ suspension of $\left[\mathrm{PtCl}_{2}\left(\mathrm{\eta}^{2}-\mathrm{coe}\right)\right]_{2}(602 \mathrm{mg}, 0.80 \mathrm{mmol})$ was added and the mixture was stirred at RT for $18 \mathrm{~h}$. Upon removing the reaction from the inert atmosphere (except 7), the suspension was passed through a plug of alumina until the solution was clear orange. Removal of solvent under vacuum afforded the crude mono-Schiff base platinum complexes. Further purification for each compound, if necessary, is described below.

(Chlorido)(n²-cis-cyclooctene)(2-((isopentylimino)methyl)phenolato- $\mathbf{k}^{2}$ -

\section{N,O)platinum(II) (1)}


Recrystallization from $\mathrm{CH}_{2} \mathrm{Cl}_{2}$ /hexane solution (5 mL: $2 \mathrm{~mL}$ ) afforded $\mathbf{1}$ as a yelloworange solid. Yield: $705 \mathrm{mg}(83 \%)$; mp: 52-54ㄷ․ ${ }^{1} \mathrm{H} \mathrm{NMR}\left(\mathrm{CDCl}_{3}\right): \delta 7.89\left(\mathrm{~s}, J_{\mathrm{HPt}}=66.8\right.$ $\mathrm{Hz}, 1 \mathrm{H}, \mathrm{C}(H)=\mathrm{N}), 7.32\left(\mathrm{ddd}, J_{\mathrm{HH}}=8.4,6.9,2.0 \mathrm{~Hz}, 1 \mathrm{H}, \mathrm{Ar}\right), 7.23\left(\mathrm{dd}, J_{\mathrm{HH}}=7.9,2.0 \mathrm{~Hz}\right.$, $1 \mathrm{H}, \mathrm{Ar}), 6.84\left(\mathrm{~d}, J_{\mathrm{HH}}=8.4 \mathrm{~Hz}, 1 \mathrm{H}, \mathrm{Ar}\right), 6.63\left(\mathrm{ddd}, J_{\mathrm{HH}}=7.9,6.9,1.0 \mathrm{~Hz}, 1 \mathrm{H}, \mathrm{Ar}\right), 5.47$ (br $\left.\mathrm{m}, J_{\mathrm{HPt}}=60.4 \mathrm{~Hz}, 2 \mathrm{H}, \mathrm{HC}=\mathrm{CH}\right), 4.18\left(\mathrm{t}, J_{\mathrm{HH}}=6.9 \mathrm{~Hz}, 2 \mathrm{H}, \mathrm{NCH}\right), 2.40(\mathrm{~m}, 2 \mathrm{H}, \mathrm{CH}$ (coe)), $2.28\left(\mathrm{~m}, 2 \mathrm{H}, \mathrm{CH}_{2}\right.$ (coe)), $1.80\left(\mathrm{~m}, 2 \mathrm{H}, \mathrm{CH}_{2}\right.$ (coe)), 1.74-1.62 (ov m, 3H, $\mathrm{CH}_{2} \& \mathrm{CH}$ (isopentyl)), 1.54-1.44 (ov m, 6H, $\left.\mathrm{CH}_{2}(\mathrm{coe})\right), 0.94\left(\mathrm{~d}, \mathrm{~J}_{\mathrm{HH}}=5.9 \mathrm{~Hz}, 6 \mathrm{H}, \mathrm{CH}\left(\mathrm{CH}_{3}\right)_{2}\right) .{ }^{13} \mathrm{C}\left\{{ }^{1} \mathrm{H}\right\}$ $\operatorname{NMR}\left(\mathrm{CDCl}_{3}\right): \delta 163.1,160.9,135.5,134.2,119.9,119.5,116.7,94.2\left(\mathrm{~s}, J_{\mathrm{CPt}}=167.1 \mathrm{~Hz}\right.$,

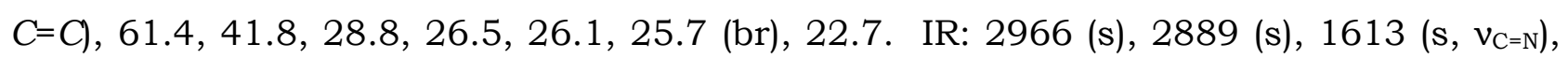
$1540(\mathrm{~m}), 1469(\mathrm{~m}), 1402$ (m), 1329 (m), 1151 (m), 1019 (m), 911 (m), 752 (m). Anal. calcd. for $\mathrm{C}_{20} \mathrm{H}_{30} \mathrm{NClOPt}$ (530.99) (\%): C 45.24, H 5.69, N 2.64. Found: C 45.40, H 5.84, N 2.43.

\section{(Chlorido)(n²-cis-cyclooctene)(2-((hexylimino)methyl)phenolato- $\left.\mathbf{k}^{2}-\mathrm{N}, 0\right)$ platinum(II)}

\section{(2)}

Orange oil. Yield: $759 \mathrm{mg}(87 \%) .{ }^{1} \mathrm{H} \mathrm{NMR}\left(\mathrm{CDCl}_{3}\right): \delta 7.87\left(\mathrm{~s}, J_{\mathrm{HPt}}=65.8 \mathrm{~Hz}, 1 \mathrm{H}, \mathrm{C}(H)=\mathrm{N}\right)$, $7.31\left(\mathrm{ddd}, J_{\mathrm{HH}}=8.4,6.9,2.0 \mathrm{~Hz}, 1 \mathrm{H}, \mathrm{Ar}\right), 7.24\left(\mathrm{dd}, J_{\mathrm{HH}}=7.9,2.0 \mathrm{~Hz}, 1 \mathrm{H}, \mathrm{Ar}\right), 6.84\left(\mathrm{~d}, J_{\mathrm{HH}}\right.$ $=8.4 \mathrm{~Hz}, 1 \mathrm{H}, \mathrm{Ar}), 6.62\left(\mathrm{ddd}, J_{\mathrm{HH}}=7.9,6.9,1.0 \mathrm{~Hz}, 1 \mathrm{H}, \mathrm{Ar}\right), 5.46\left(\mathrm{br} \mathrm{m}, J_{\mathrm{HPt}}=63.3 \mathrm{~Hz}\right.$, $2 \mathrm{H}, \mathrm{HC}=\mathrm{CH}), 4.14\left(\mathrm{t}, \mathrm{J}_{\mathrm{HH}}=7.4 \mathrm{~Hz}, 2 \mathrm{H}, \mathrm{NCH}_{2}\right), 2.46\left(\mathrm{~m}, 2 \mathrm{H}, \mathrm{CH}_{2}(\mathrm{coe})\right), 2.25\left(\mathrm{~m}, 2 \mathrm{H}, \mathrm{CH}_{2}\right.$ (coe)), 1.90-1.74 (br m, 4H, $\mathrm{CH}_{2}$ (hexyl \& coe)), 1.56-1.41 (ov m, 6H, CH (coe)), 1.31 (br $\mathrm{m}, 6 \mathrm{H}, \mathrm{CH}_{2}\left(\right.$ hexyl)), $0.84\left(\mathrm{t}, \mathrm{J}_{\mathrm{HH}}=6.2 \mathrm{~Hz}, 3 \mathrm{H}, \mathrm{CH} \mathrm{H}_{3}\right) .{ }^{13} \mathrm{C}\left\{{ }^{1} \mathrm{H}\right\} \mathrm{NMR}\left(\mathrm{CDCl}_{3}\right): \delta$ 163.0, 160.7, 135.3, 134.0, 119.7, 119.2, 116.5, $94.0\left(\mathrm{~s}, J_{\mathrm{CPt}}=160.9 \mathrm{~Hz}, C=C\right), 62.8,32.5,31.4,28.6$, 26.3, 26.1, 25.5 (br), 22.5, 14.0. IR: $3027(\mathrm{~m}), 2908(\mathrm{~m}), 1613\left(\mathrm{~s}, \mathrm{v}_{\mathrm{C}=\mathrm{N}}\right), 1540(\mathrm{~m}), 1449$ (m), $1328(\mathrm{~m}), 1151$ (m), $1024(\mathrm{~m}), 914(\mathrm{~m}), 752$ (m). Anal. calcd. for $\mathrm{C}_{21} \mathrm{H}_{32} \mathrm{NClOPt}$ (545.02) (\%): C 46.28, H 5.92, N 2.57. Found: C 46.49, H 6.01, N 2.40. 


\section{(Chlorido)(n²-cis-cyclooctene)(2-((octylimino)methyl)phenolato-k $\left.{ }^{2}-\mathrm{N}, 0\right)$ platinum(II)}

(3)

Orange oil. Yield: $761 \mathrm{mg}(83 \%) .{ }^{1} \mathrm{H} \mathrm{NMR}\left(\mathrm{CDCl}_{3}\right): \delta 7.87\left(\mathrm{~s}, J_{\mathrm{HPt}}=65.3 \mathrm{~Hz}, 1 \mathrm{H}, \mathrm{C}(H)=\mathrm{N}\right)$, $7.32\left(\mathrm{ddd}, J_{\mathrm{HH}}=8.4,6.9,1.7 \mathrm{~Hz}, 1 \mathrm{H}, \mathrm{Ar}\right), 7.24\left(\mathrm{dd}, J_{\mathrm{HH}}=7.9,1.7 \mathrm{~Hz}, 1 \mathrm{H}, \mathrm{Ar}\right), 6.84\left(\mathrm{~d}, J_{\mathrm{HH}}\right.$ $=8.4 \mathrm{~Hz}, 1 \mathrm{H}, \mathrm{Ar}), 6.63\left(\mathrm{ddd}, J_{\mathrm{HH}}=7.9,6.9,1.0 \mathrm{~Hz}, 1 \mathrm{H}, \mathrm{Ar}\right), 5.47\left(\mathrm{br} \mathrm{m}, J_{\mathrm{HPt}}=60.8 \mathrm{~Hz}\right.$, $2 \mathrm{H}, \mathrm{HC}=\mathrm{CH}), 4.14\left(\mathrm{t}, J_{\mathrm{HH}}=7.4 \mathrm{~Hz}, 2 \mathrm{H}, \mathrm{NCH}_{2}\right), 2.46\left(\mathrm{~m}, 2 \mathrm{H}, \mathrm{CH}_{2}(\mathrm{coe})\right), 2.28\left(\mathrm{~m}, 2 \mathrm{H}, \mathrm{CH}_{2}\right.$ (coe)), 1.90-1.76 (br m, 4H, $\mathrm{CH}_{2}$ (octyl \& coe)), 1.56-1.41 (ov m, 6H, CH (ov m, 10H, $\mathrm{CH}_{2}$ (octyl)), 0.86 (t, $\left.J_{\mathrm{HH}}=6.9 \mathrm{~Hz}, 3 \mathrm{H}, \mathrm{CH}_{3}\right) .{ }^{13} \mathrm{C}\left\{{ }^{1} \mathrm{H}\right\} \mathrm{NMR}\left(\mathrm{CDCl}_{3}\right): \delta 163.1$, $160.9,135.5,134.3,119.9,119.4,116.7,94.2\left(\mathrm{~s}, J_{\mathrm{CPt}}=156.6 \mathrm{~Hz}, C=C\right), 63.0,32.8,31.9$, 29.4, 29.3, 28.8, 26.6, 26.5, $25.7(\mathrm{br}), 22.7,14.2$. IR: $2924(\mathrm{~m}), 2851(\mathrm{~m}), 1613\left(\mathrm{~s}, \mathrm{v}_{\mathrm{C}=\mathrm{N}}\right)$, 1541 (m), 1449 (m), 1328 (s), 1204 (m), 1151 (m), 1025 (m), 912 (m), 753 (m). Anal. calcd. for $\mathrm{C}_{23} \mathrm{H}_{36} \mathrm{NClOPt}$ (573.07) (\%): C 48.20, H 6.33, N 2.44. Found: C 48.16, H 6.61, N 2.22.

\section{(Chlorido)(n²-cis-cyclooctene)(2-((phenylimino)methyl)phenolato-k $\left.{ }^{2}-\mathbf{N}, 0\right)$ platinum(II)}

\section{(4)}

Recrystallization from a $\mathrm{CH}_{2} \mathrm{Cl}_{2}$ /hexane solution (5 mL: $2 \mathrm{~mL}$ ) afforded 4 as an orange solid. Yield: $715 \mathrm{mg}(78 \%)$; mp: 164-166 ${ }^{\circ} \mathrm{C} .{ }^{1} \mathrm{H} \mathrm{NMR}\left(\mathrm{CDCl}_{3}\right): \delta 8.06$ (s, $J_{\mathrm{HPt}}=62.0 \mathrm{~Hz}$, $1 \mathrm{H}, \mathrm{C}(H)=\mathrm{N}), 7.43-7.37$ (ov m, 3H, Ar), 7.28 (dd, $\left.J_{\mathrm{HH}}=7.7,1.5 \mathrm{~Hz}, 1 \mathrm{H}, \mathrm{Ar}\right), 7.25-7.21$ (ov $\mathrm{m}, 3 \mathrm{H}, \mathrm{Ar}), 6.92\left(\mathrm{~d}, J_{\mathrm{HH}}=8.4 \mathrm{~Hz}, 1 \mathrm{H}, \mathrm{Ar}\right), 6.67\left(\mathrm{ddd}, J_{\mathrm{HH}}=7.7,6.9,1.0 \mathrm{~Hz}, 1 \mathrm{H}, \operatorname{Ar}\right), 5.50$ (br m, J JPt $=60.9 \mathrm{~Hz}, 2 \mathrm{H}, \mathrm{HC}=\mathrm{CH}), 2.50\left(\mathrm{~m}, 2 \mathrm{H}, \mathrm{CH} \mathrm{H}_{2}\right), 2.30\left(\mathrm{~m}, 2 \mathrm{H}, \mathrm{CH}_{2}\right), 1.81(\mathrm{br} \mathrm{m}, 2 \mathrm{H}$, $\left.\mathrm{CH}_{2}\right), 1.57-1.49$ (ov m, 6H, $\left.\mathrm{CH}_{2}\right) .{ }^{13} \mathrm{C}\{1 \mathrm{H}\} \mathrm{NMR}\left(\mathrm{CDCl}_{3}\right): \delta 164.0,161.8,152.0,136.5$, 134.9, 128.5, 127.1, 124.6, 120.6, 119.1, 117.1, $94.0\left(\mathrm{~s}, J_{\mathrm{CPt}}=167.6 \mathrm{~Hz}, C=C\right), 28.8$, 26.4, 25.8. IR: $2926(\mathrm{~s}), 2856(\mathrm{~m}), 1610\left(\mathrm{~s}, \mathrm{v}_{\mathrm{C}=\mathrm{N}}\right), 1536(\mathrm{~m}), 1466(\mathrm{~s}), 1382(\mathrm{~m}), 1316(\mathrm{~m})$, 
1183 (m), 755 (m). Anal. calcd. for $\mathrm{C}_{21} \mathrm{H}_{24} \mathrm{NClOPt} \cdot \mathrm{CH}_{2} \mathrm{Cl}_{2}$ (621.88) (\%): C 42.49, H 4.21, N 2.25. Found: C 42.29, H 3.94, N 2.06.

(Chlorido)(n²-cis-cyclooctene)(2-((4-methoxyphenylimino)methyl)phenolato-k²$\mathrm{N}$, O)platinum(II) (5)

Recrystallization from a $\mathrm{Et}_{2} \mathrm{O} /$ hexane $(10 \mathrm{~mL}$ : $5 \mathrm{~mL})$ solution afforded $\mathbf{5}$ as a yelloworange solid. Yield: $689 \mathrm{mg}(76 \%)$; mp: 131-133॰C. ${ }^{1} \mathrm{H}$ NMR $\left(\mathrm{CDCl}_{3}\right): \delta 8.03\left(\mathrm{~s}, J_{\mathrm{HPt}}=\right.$ $60.9 \mathrm{~Hz}, 1 \mathrm{H}, \mathrm{C}(H)=\mathrm{N}), 7.38\left(\mathrm{ddd}, J_{\mathrm{HH}}=8.4,6.8,1.8 \mathrm{~Hz}, 1 \mathrm{H}, \mathrm{Ar}\right), 7.26\left(\mathrm{dd}, J_{\mathrm{HH}}=7.7,1.8\right.$ $\mathrm{Hz}, 1 \mathrm{H}, \mathrm{Ar}), 7.16\left(\mathrm{~d}, J_{\mathrm{HH}}=8.9 \mathrm{~Hz}, 2 \mathrm{H}, \mathrm{Ar}\right), 6.90\left(\mathrm{~d}, J_{\mathrm{HH}}=8.9 \mathrm{~Hz}, 2 \mathrm{H}, \mathrm{Ar}\right), 6.88\left(\mathrm{~d}, J_{\mathrm{HH}}=\right.$ $8.4 \mathrm{~Hz}, 1 \mathrm{H}, \mathrm{Ar}), 6.66\left(\mathrm{ddd}, J_{\mathrm{HH}}=7.7,6.8,1.0 \mathrm{~Hz}, 1 \mathrm{H}, \mathrm{Ar}\right), 5.49\left(\mathrm{br} \mathrm{m}, J_{\mathrm{HPt}}=58.9 \mathrm{~Hz}, 2 \mathrm{H}\right.$, $H \mathrm{C}=\mathrm{CH}), 3.81\left(\mathrm{~s}, 3 \mathrm{H}, \mathrm{OCH}_{3}\right), 2.50\left(\mathrm{~m}, 2 \mathrm{H}, \mathrm{CH}_{2}\right), 2.30\left(\mathrm{~m}, 2 \mathrm{H}, \mathrm{CH}_{2}\right), 1.81\left(\mathrm{br} \mathrm{m}, 2 \mathrm{H}, \mathrm{CH}_{2}\right)$, 1.53-1.49 (ov m, 6H, CH$\left.H_{2}\right) .{ }^{13} \mathrm{C}\left\{{ }^{1} \mathrm{H}\right\} \operatorname{NMR}\left(\mathrm{CDCl}_{3}\right): \delta 163.9,161.8,158.3,145.4,136.4$, $134.8,125.5,120.5,119.2,117.1,113.5,94.0\left(\mathrm{~s}, J_{\mathrm{CPt}}=168.3 \mathrm{~Hz}, C=C\right), 55.5,28.8,26.4$, 25.8. IR: $2915(\mathrm{~m}), 2848(\mathrm{~m}), 1613\left(\mathrm{~s}, \mathrm{v}_{\mathrm{C}=\mathrm{N}}\right), 1504(\mathrm{~m}), 1465(\mathrm{~m}), 1299(\mathrm{~m}), 1244(\mathrm{~m})$, $1182(\mathrm{~m}), 827$ (m), $762(\mathrm{~m})$. Anal. calcd. for $\mathrm{C}_{22} \mathrm{H}_{26} \mathrm{NClO}_{2} \mathrm{Pt}$ (566.98) (\%): C 46.60, H 4.62, N 2.47. Found: C 46.42, H 4.38, N 2.59.

(Chlorido)(n²-cis-cyclooctene)(2-((4-fluorophenylimino)methyl)phenolato-k2 ${ }^{2}$ N,O)platinum(II) (6)

Recrystallization from a $\mathrm{CH}_{2} \mathrm{Cl}_{2}$ /hexane $(10 \mathrm{~mL}$ : $5 \mathrm{~mL}$ ) solution afforded 6 as a yellow solid. Yield: $746 \mathrm{mg}(84 \%)$; mp: 156-158 ${ }^{\circ} \mathrm{C} .{ }^{1} \mathrm{H}$ NMR $\left(\mathrm{CDCl}_{3}\right): \delta 8.05$ (s, $J_{\mathrm{HPt}}=61.4 \mathrm{~Hz}$, $1 \mathrm{H}, \mathrm{C}(H)=\mathrm{N}), 7.41\left(\mathrm{ddd}, J_{\mathrm{HH}}=8.4,6.9,2.0 \mathrm{~Hz}, 1 \mathrm{H}, \mathrm{Ar}\right), 7.28\left(\mathrm{dd}, J_{\mathrm{HH}}=7.9,2.0 \mathrm{~Hz}, 1 \mathrm{H}\right.$, Ar), 7.21-7.15 (ov m, 2H, Ar), 7.05 (m, 2H, Ar), 6.92 (d, $\left.J_{\mathrm{HH}}=8.4 \mathrm{~Hz}, 1 \mathrm{H}, \mathrm{Ar}\right), 6.67$ (ddd, $\left.J_{\mathrm{HH}}=7.9,6.9,1.0 \mathrm{~Hz}, 1 \mathrm{H}, \mathrm{Ar}\right), 5.50\left(\mathrm{br} \mathrm{m}, J_{\mathrm{HPt}}=61.9 \mathrm{~Hz}, 2 \mathrm{H}, H \mathrm{C}=\mathrm{CH}\right), 2.50(\mathrm{~m}, 2 \mathrm{H}$, $\left.\mathrm{CH}_{2}\right), 2.30\left(\mathrm{~m}, 2 \mathrm{H}, \mathrm{CH}_{2}\right), 1.81$ (br m, 2H, $\left.\mathrm{CH}_{2}\right), 1.53-1.49$ (ov m, 6H, CH$\left.H_{2}\right) .{ }^{13} \mathrm{C}\left\{{ }^{1} \mathrm{H}\right\} \mathrm{NMR}$ 
$\left(\mathrm{CDCl}_{3}\right): \delta 164.1,162.0,161.3\left(\mathrm{~d}, J_{\mathrm{CF}}=246.0 \mathrm{~Hz}, \mathrm{CF}\right), 148.0\left(\mathrm{~d}, J_{\mathrm{CF}}=2.6 \mathrm{~Hz}\right), 136.7$, 135.0, $126.2\left(\mathrm{~d}, J_{\mathrm{CF}}=8.3 \mathrm{~Hz}\right), 120.7,118.9,117.2,115.2\left(\mathrm{~d}, J_{\mathrm{CF}}=22.8 \mathrm{~Hz}\right), 94.2\left(\mathrm{~s}, J_{\mathrm{CPt}}\right.$ $=153.6 \mathrm{~Hz}, C=C), 28.8,26.4,25.9 .{ }^{19} \mathrm{~F}\left\{{ }^{1} \mathrm{H}\right\} \mathrm{NMR}\left(\mathrm{CDCl}_{3}\right): \delta=-115.4 . \quad$ IR: $2976(\mathrm{~m}), 2881$ $(\mathrm{m}), 2841(\mathrm{~m}), 1609\left(\mathrm{~s}, \mathrm{v}_{\mathrm{C}=\mathrm{N}}\right), 1589(\mathrm{~m}), 1535(\mathrm{~m}), 1441(\mathrm{~m}), 1382(\mathrm{~m}), 1316(\mathrm{~m}), 1181$ (m), $844(\mathrm{~m}), 752$ (m). Anal. calcd. for $\mathrm{C}_{21} \mathrm{H}_{23} \mathrm{NClFOPt}$ (554.94) (\%): C 45.45, H 4.18, N 2.52. Found: C 45.40, H 4.31, N 2.29.

\section{(Chlorido)(n²-cis-cyclooctene)(2-((4-(4,4,5,5-tetramethyl-1,3,2-dioxaborolan-2-}

\section{yl)phenylimino)methyl)phenolato-k $\left.{ }^{2}-\mathrm{N}, 0\right)$ platinum(II) (7)}

This compound is air-sensitive therefore all manipulations were performed under an atmosphere of dinitrogen. Slow evaporation of a hexane $(10 \mathrm{~mL})$ solution afforded $\mathbf{7}$ as a yellow-orange crystalline solid. Yield: $573 \mathrm{mg}(54 \%)$; mp: 192-194ㅇ․ ${ }^{1} \mathrm{H} \mathrm{NMR}\left(\mathrm{CDCl}_{3}\right): \delta$ $8.01\left(\mathrm{~s}, J_{\mathrm{HPt}}=55.0 \mathrm{~Hz}, 1 \mathrm{H}, \mathrm{C}(H)=\mathrm{N}\right), 7.84\left(\mathrm{~d}, J_{\mathrm{HH}}=8.2 \mathrm{~Hz}, 2 \mathrm{H}, \mathrm{Ar}\right), 7.40\left(\mathrm{ddd}, J_{\mathrm{HH}}=8.2\right.$, 6.8, $1.4 \mathrm{~Hz}, 1 \mathrm{H}, \mathrm{Ar}$ ), 7.27-7.23 (ov m, 3H, Ar), 6.91 (d, $J=8.2 \mathrm{~Hz}, 1 \mathrm{H}, \mathrm{Ar}$ ), 6.66 (ov dd, $\left.J_{\mathrm{HH}}=7.8,6.9 \mathrm{~Hz}, 1 \mathrm{H}, \mathrm{Ar}\right), 5.51\left(\mathrm{br} \mathrm{m}, J_{\mathrm{HPt}}=62.8 \mathrm{~Hz}, 2 \mathrm{H}, H \mathrm{C}=\mathrm{CH}\right), 2.51\left(\mathrm{~m}, 2 \mathrm{H}, \mathrm{C} H_{2}\right)$, $2.30\left(\mathrm{~m}, 2 \mathrm{H}, \mathrm{CH}_{2}\right.$ ), 1.83 (br m, 2H, CH$H_{2}$, 1.53-1.49 (ov m, 6H, CH $\left.H_{2}\right), 1.33$ (s, $12 \mathrm{H}, \mathrm{CH}_{3}$ ). ${ }^{11} \mathrm{~B}$ NMR $\left(\mathrm{CDCl}_{3}\right): \delta 30(\mathrm{br}) .{ }^{13} \mathrm{C}\left\{{ }^{1} \mathrm{H}\right\} \mathrm{NMR}\left(\mathrm{CDCl}_{3}\right): \delta 164.0,161.9,151.5,135.0,133.6$, 130 (br, CB), 129.6, 128.4, 120.5, 119.1, $117.0,93.9\left(\mathrm{~s}, J_{\mathrm{CPt}}=161.1 \mathrm{~Hz}, C=C\right), 84.1$, 28.8, 26.5, 25.8, 25.0. IR: $2986(\mathrm{~m}), 2876(\mathrm{~m}), 2839(\mathrm{~m}), 1615\left(\mathrm{~s}, \mathrm{v}_{\mathrm{C}=\mathrm{N}}\right), 1588(\mathrm{~m}), 1532$ (m), 1395 (m), 1309 (m), 1183 (m), 748 (m). Anal. calcd. for $\mathrm{C}_{27} \mathrm{H}_{35} \mathrm{NBClO}_{3} \mathrm{Pt}$ (662.92) (\%): C 48.92, H 5.32, N 2.11. Found: C 48.60, H 5.31, N 2.39.

\section{Stability testing of platinum complexes in the presence of water}

In an NMR tube, compound 5 was dissolved in $\mathrm{CDCl}_{3}(1 \mathrm{~mL})$ and $\mathrm{D}_{2} \mathrm{O}(3$ drops $)$ was added. The mixture was analyzed by ${ }^{1} \mathrm{H}$ NMR spectroscopy after $1,2,4$, and $24 \mathrm{~h}$. 


\section{X-ray crystallography}

Crystals of $\mathbf{4}$ were grown from a saturated toluene solution stored at $5^{\circ} \mathrm{C}$. Crystals of $\mathbf{7}$ were grown by slow evaporation of a hexane solution at RT under an inert atmosphere. Single crystals were coated with Paratone-N oil, mounted using a polyimide MicroMount, and frozen in the cold nitrogen stream of the goniometer. Hemispheres of data were collected on a Bruker AXS P4/SMART 1000 diffractometer using $\omega$ and $\phi$ scans with a scan width of $0.3^{\circ}$ and $10 \mathrm{~s}$ exposure times. The detector distance was $5 \mathrm{~cm}$. The crystal of 4 was twinned and the orientation matrixes for two components were determined (CELL_NOW) ${ }^{13}$ and integration of the data resulted in $94 \%$ completeness. The data were reduced (SAINT) ${ }^{14}$ and corrected for absorption (TWINABS). ${ }^{15}$ The structure was solved by direct methods and refined by full-matrix least squares on $\mathrm{F}^{2}$ (SHELXTL) ${ }^{16}$ on all data. The coe ligand was disordered and the site occupancy determined using an isotropic model as $0.53(\mathrm{C}(17)-\mathrm{C}(20))$ and $0.47(\mathrm{C}(17 \mathrm{~A})-\mathrm{C}(20 \mathrm{~A}))$ and fixed in subsequent refinement cycles. The data for $\mathbf{7}$ were reduced (SAINT) and corrected for absorption (SADABS). ${ }^{17}$ The structure was solved by direct methods and refined by full-matrix least squares on $\mathrm{F}^{2}$ (SHELXTL). The coe ligand was disordered over two positions and the site occupancies determined using an isotropic model as 0.55 $(\mathrm{C}(24)-\mathrm{C}(25))$ and $0.45(\mathrm{C}(24 \mathrm{~A})-\mathrm{C}(25 \mathrm{~A}))$ and fixed in subsequent refinement cycles. The Bpin group was disordered over two positions and the site occupancies determined using an isotropic model as $0.75(\mathrm{C}(16)-\mathrm{C}(17))$ and $0.25(\mathrm{C}(16 \mathrm{~A})-\mathrm{C}(17 \mathrm{~A}))$ and fixed in subsequent refinement cycles. Bond lengths within the disordered parts were restrained to equal values and atoms in close proximities were constrained to the same thermal parameters. For $\mathbf{4}$ and $\mathbf{7}$, all non-hydrogen atoms were refined using anisotropic 
displacement parameters and hydrogen atoms were included in calculated positions and refined using a riding model.

\section{Cell cultures and biological testing}

The MDA-MB-231 (MB231) ductal carcinoma mammary cell line (HTB-26) was obtained from the American Type Culture Collection (ATCC, Manassas, VA, USA). RCC4, RCC4/VHL, RCC10, RCC10/VHL, 786-0 and 786-0/VHL were a gift from Amato J. Giaccia at Stanford University. Cells were cultured in DMEM medium supplemented with 10\% foetal bovine serum (FBS), L-glutamine (2 mM), penicillin (100 units $/ \mathrm{mL})$, and streptomycin $(100 \mu \mathrm{g} / \mathrm{mL})$. Culture media and reagents were obtained from Life Technologies (Burlington, ON, Canada) except for the FBS which was provided by PAA Laboratories (ON, Canada). Primary renal proximal tubule epithelial cells (RPTEC) obtained from ATCC and were cultured in renal epithelial growth media (REGM) from Lonza (Cedarlanelabs, Burlington, ON, Canada).

Biological assays were performed with $5 \times 10^{3}$ cells seeded in 96 well plates and analyzed at the indicated time points for cellular viability and apoptosis using a fluorescent-based assays with CellTiter Blue ${ }^{\circledR}$ and Apo-ONE ${ }^{\circledR}$ reagents (Promega, Madison, WI, USA). In brief, $20 \mu \mathrm{L}$ of CellTiter Blue ${ }^{\circledR}$ substrate was added to $100 \mu \mathrm{L}$ of media containing the cells and incubated at $37^{\circ} \mathrm{C}$ for 1 hour. Platinum complexes were dissolved in DMSO at a stock concentration of $1 \mathrm{mM}$. The plates were analyzed on a fluorescence microplate reader (FLUOstar Optima, BMG Lab technologies, 544 $\mathrm{Ex} / 590_{\mathrm{Em}}$ ). Apoptosis was also measured on the same microplate by removing $80 \mu \mathrm{L}$ of the total media and adding $40 \mu \mathrm{L}$ of the Apo-ONE® substrate. Plates were next incubated at room temperature for 1 hour on a plate shaker and analyzed by fluorescence reading $\left(485_{\mathrm{Ex}} / 520_{\mathrm{Em}}\right)$. 


\section{Results and discussion}

We have prepared a series of organometallic (salicylaldiminato)platinum complexes by the addition of lithiated salicylaldimines to the dimer $\left[\mathrm{PtCl}_{2}\left(\eta^{2}-\mathrm{coe}\right)\right]_{2}$ (coe $=$ ciscyclooctene). The resulting cis-chlorido(cis-cyclooctene)(salicylaldiminato)platinum(II) complexes 1-7 were isolated in good yields (Scheme 1) and characterized by multinuclear NMR and FT-IR spectroscopy as well as elemental analyses. Coordination of the Schiff base ligands is confirmed by ${ }^{1} \mathrm{H}$ NMR spectroscopy as the $\mathrm{sp}^{2}$ imine proton shifts upfield from ca. 9 ppm for the free ligands to ca. $8 \mathrm{ppm}$ for the metal complexes. Furthermore, the phenolic hydrogen in the uncoordinated ligand, observed at around $13.5 \mathrm{ppm}$, disappears upon complexation. The platinum satellites for the imine resonances $\left(J_{\mathrm{HPt}} \sim 62 \mathrm{~Hz}\right)$ are consistent with other (salicylaldiminato)platinum complexes. ${ }^{18-26}$ The FT-IR spectra show the expected decrease in the frequency of the $\mathrm{C}=\mathrm{N}$ bond due to complexation, which is observed as the $\mathrm{v}_{\mathrm{C}=\mathrm{N}}$ stretch shifts from $c a$. $1632 \mathrm{~cm}^{-1}$ for the free ligands to $\mathrm{ca} .1614 \mathrm{~cm}^{-1}$ for the platinum compounds. ${ }^{12}$ As well, the absence of the $v_{\mathrm{OH}}$ shift in the metal complexes, observed at $c a .2860 \mathrm{~cm}^{-1}$ for the free Schiff bases, further confirms coordination of these bidentate ligands. As seen by multinuclear NMR spectroscopy, the cyclooctene ligand remains coordinated to the platinum centre as the ${ }^{1} \mathrm{H}$ NMR data show a broad multiplet at ca. $5.5 \mathrm{ppm}$ for the bound alkene hydrogens along with coupling to platinum $\left(J_{\mathrm{HPt}}=60 \mathrm{MHz}\right)$. The platinum bound olefinic carbons show up at $\delta 94 \mathrm{ppm}$ in the ${ }^{13} \mathrm{C}\left\{{ }^{1} \mathrm{H}\right\}$ NMR spectra with platinum coupling of ca. $J_{\mathrm{CPt}}=162 \mathrm{~Hz} \cdot{ }^{11}$ Elemental analyses are fully consistent with the mono Schiff base formulation. 
Complexes 4 and $\mathbf{7}$ have also been characterized by single crystal X-ray diffraction studies, the molecular structure of both square planar complexes are shown in Figure 2 and relevant crystal data are given in Table 1 . In both structures, the nitrogen is trans to the alkene group of cyclooctene. The imine carbon-nitrogen bond lengths are $1.313(11) \AA(4)$ and $1.300(5) \AA(7)$, respectively, suggesting significant double bond character. In comparison, the related C-N single bonds from the amine group (C8-N1) are 1.445(11) $\AA$ (4) and 1.440(4) $\AA$ (7). The bond lengths for Pt-O are 2.013(6) $\AA$ (4) and $1.996(3) \AA(7)$ and the $\mathrm{Pt}-\mathrm{N}$ bonds are 2.038(7) $\AA$ (4) and 2.056(3) $\AA$ (7). Bond distances are similar to those found in the related mono salicylaldoximato platinum complex, $\left[\mathrm{PtCl}\left\{\left(\mathrm{OC}_{6} \mathrm{H}_{4}\right) \mathrm{CH}=\mathrm{N}\left\{2,6-\left(\mathrm{Me}_{2} \mathrm{CH}\right)_{2}\left(\mathrm{C}_{6} \mathrm{H}_{3}\right)\right\}\right\}\left(\mathrm{PPh}_{3}\right)\right]$, where the $\mathrm{Pt}$ - O bond is $2.001(2) \AA$ and the $\mathrm{Pt}-\mathrm{N}$ distance is 2.0707(2) $\AA .{ }^{13}$ The $\mathrm{B}-\mathrm{O}$ bond distances of $\mathrm{B}(1)-\mathrm{O}(2) 1.348(6)$ and $\mathrm{B}(1)-\mathrm{O}(3) 1.370(6) \AA$ for the Bpin fragment (pin = pinacolato, $1,2-\mathrm{O}_{2} \mathrm{C}_{2} \mathrm{Me}_{4}$ ) in $\mathbf{7}$ are typical for those observed in other three coordinate boron compounds. ${ }^{27}$ The boron atom in 7 is observed as a broad resonance at $30 \mathrm{ppm}$ by ${ }^{11} \mathrm{~B}$ NMR spectroscopy, indicative of a three-coordinate boron environment in solution.

While compounds $\mathbf{1}-\mathbf{6}$ are insoluble, yet stable, in water, the cyclooctene group has been shown to drastically improve solubilities in common organic solvents such as $\mathrm{CHCl}_{3}, \mathrm{CH}_{2} \mathrm{Cl}_{2}$, and THF over the corresponding bis Schiff base analogues. ${ }^{7}$ Exploiting lipophilic groups to improve the delivery of platinum complexes in cancer treatment has been recently studied. ${ }^{28-30}$ In this study, compounds $\mathbf{1}-\mathbf{3}$ have been prepared from long-chain amines to enhance lipophilicity. We have also employed electronically diverse aromatic amines for complexes 4-7 in order to determine whether electron-donating (5) or electron-withdrawing groups $(\mathbf{6 , 7})$ have any effect upon the anticancer activities of the resultant platinum compounds. Platinum complexes containing only one chloride ligand 
have recently shown cytotoxic efficacy towards human ovarian carcinoma cells. ${ }^{31,32}$ Unfortunately, while boron-containing compounds are being examined for their potential bioactivites, ${ }^{33}$ complex 7 decomposes readily in air via $\mathrm{B}-\mathrm{C}$ bond cleavage and loss of the boron group to give 4 and other unidentified products, precluding biological testing of this complex.

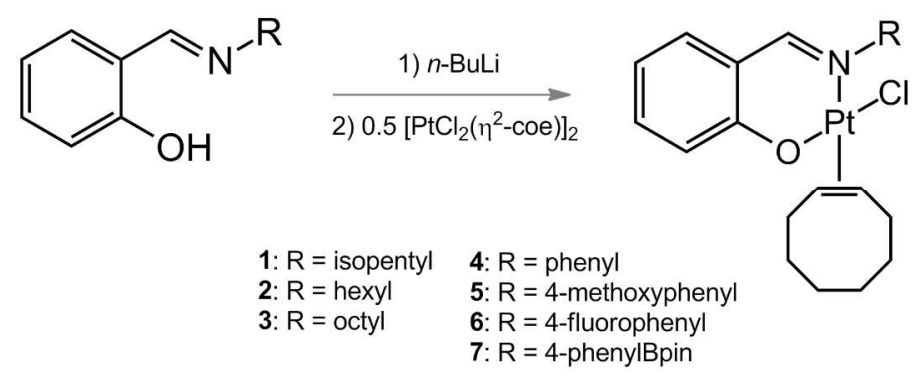

Scheme 1. Synthesis of complexes $\mathbf{1}-\mathbf{7}$.

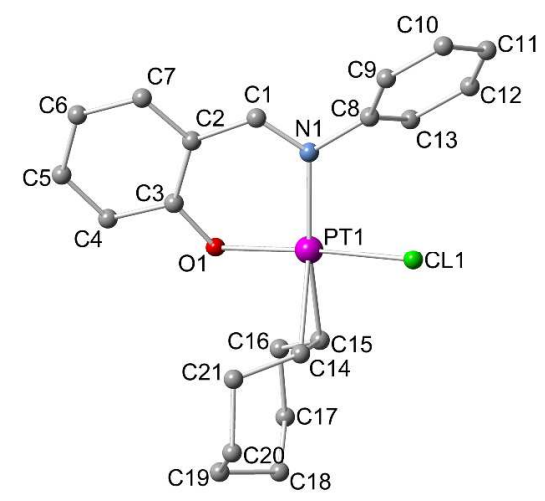

(a)

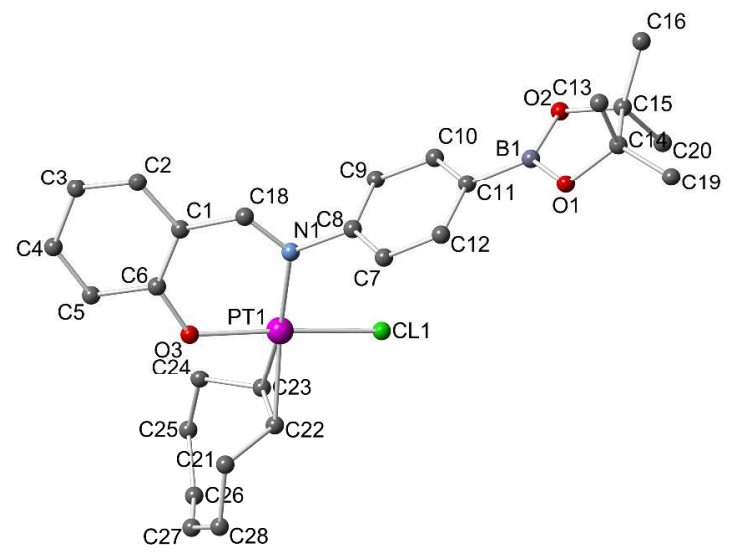

(b)

Figure 2. Ball and stick diagrams of the molecular structures of complexes 4 (a) and 7 (b) with hydrogen atoms omitted for clarity. Selected bond distances: $\mathrm{Pt}(1)-\mathrm{N}(1) 2.038(7)$, $\mathrm{Pt}(1)-\mathrm{O}(1)$ 2.013(6), Pt(1)-C(15) 2.158(8), Pt(1)-C(14) 2.180(8), Pt(1)-Cl(1) 2.301(2), C(14)$\mathrm{C}(15)$ 1.391(12) ̊̊ (4); Pt(1)-O(1) 1.996(3), Pt(1)-N(1) 2.056(3), Pt(1)-C(21) 2.170(4), Pt(1)$\mathrm{C}(20)$ 2.171(4), $\mathrm{Pt}(1)-\mathrm{Cl}(1)$ 2.3024(10), $\mathrm{N}(1)-\mathrm{C}(1)$ 1.300(5), $\mathrm{N}(1)-\mathrm{C}(8)$ 1.440(4), O(2)-B(1) $1.348(6), \mathrm{O}(3)-\mathrm{B}(1) 1.370(6) \AA ̊ ⑺$. 
In order to evaluate the potential of complexes $\mathbf{1}-\mathbf{6}$ as a source of anticancer agents, we first assessed the biological effects of the compounds on an aggressive breast cancer cell line (MB231), using cisplatin as a control.34 Using a microscale fluorometric test, MB231 viability was monitored in time (days 1,3 and 5) following the exposure of cells to $10 \mu \mathrm{M}$ of the platinum complexes. We observed that non-treated parental cells and vehicletreated (DMSO) control samples proliferated in time (Figure 3a). On the other hand, cisplatin suppressed cellular growth (approximately 62\% at day 5) when compared to parental and DMSO_treated controls. More interestingly, all novel compounds 1 to 6 inhibited up to $99 \%$ of cellular viability after only $24 \mathrm{~h}$ post-treatment. Varying the imine group in $\mathbf{1}-\mathbf{6}$ had only negligible effect in this study albeit all new complexes showed enhanced efficacy compared to cisplatin.

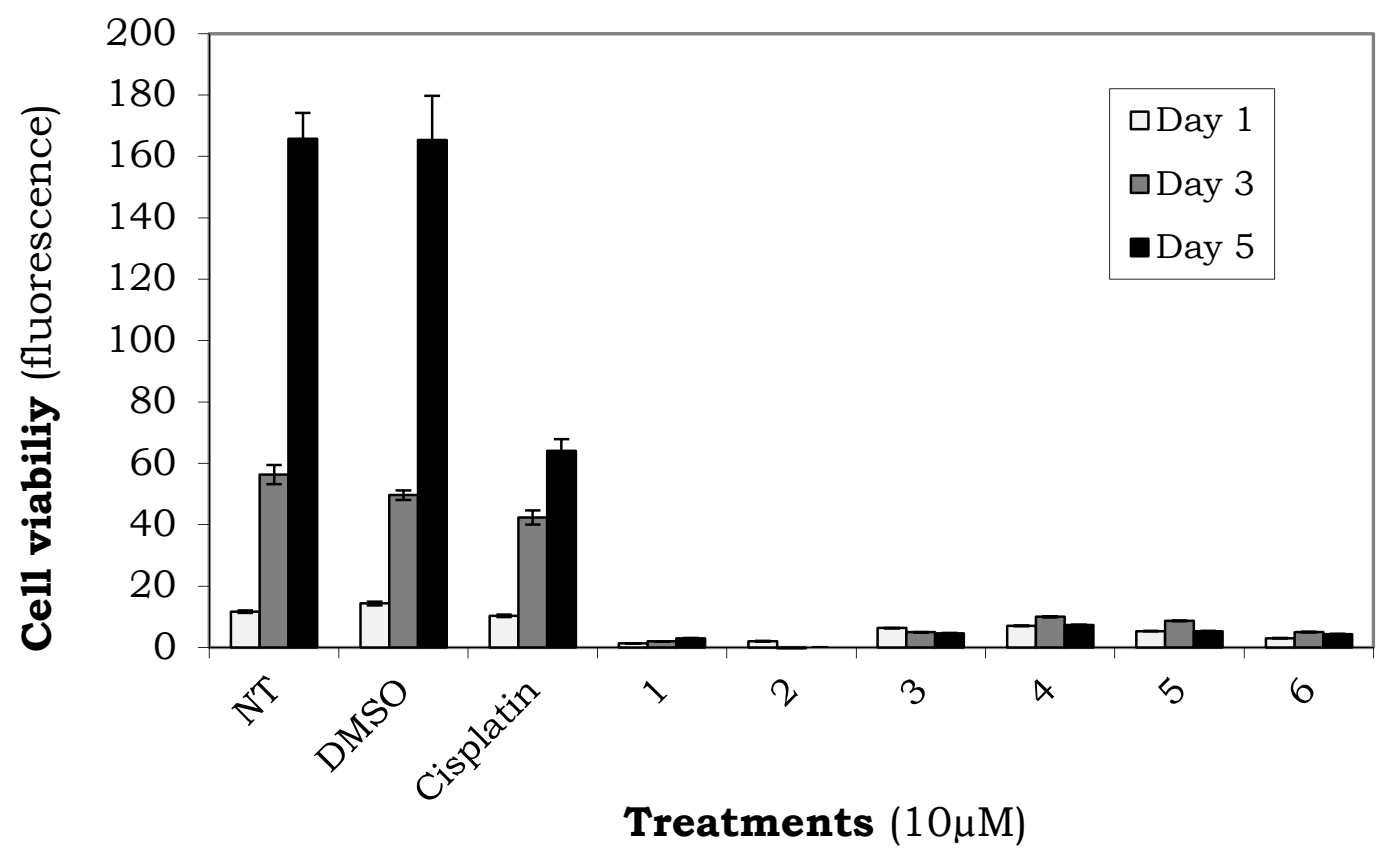

(a) 


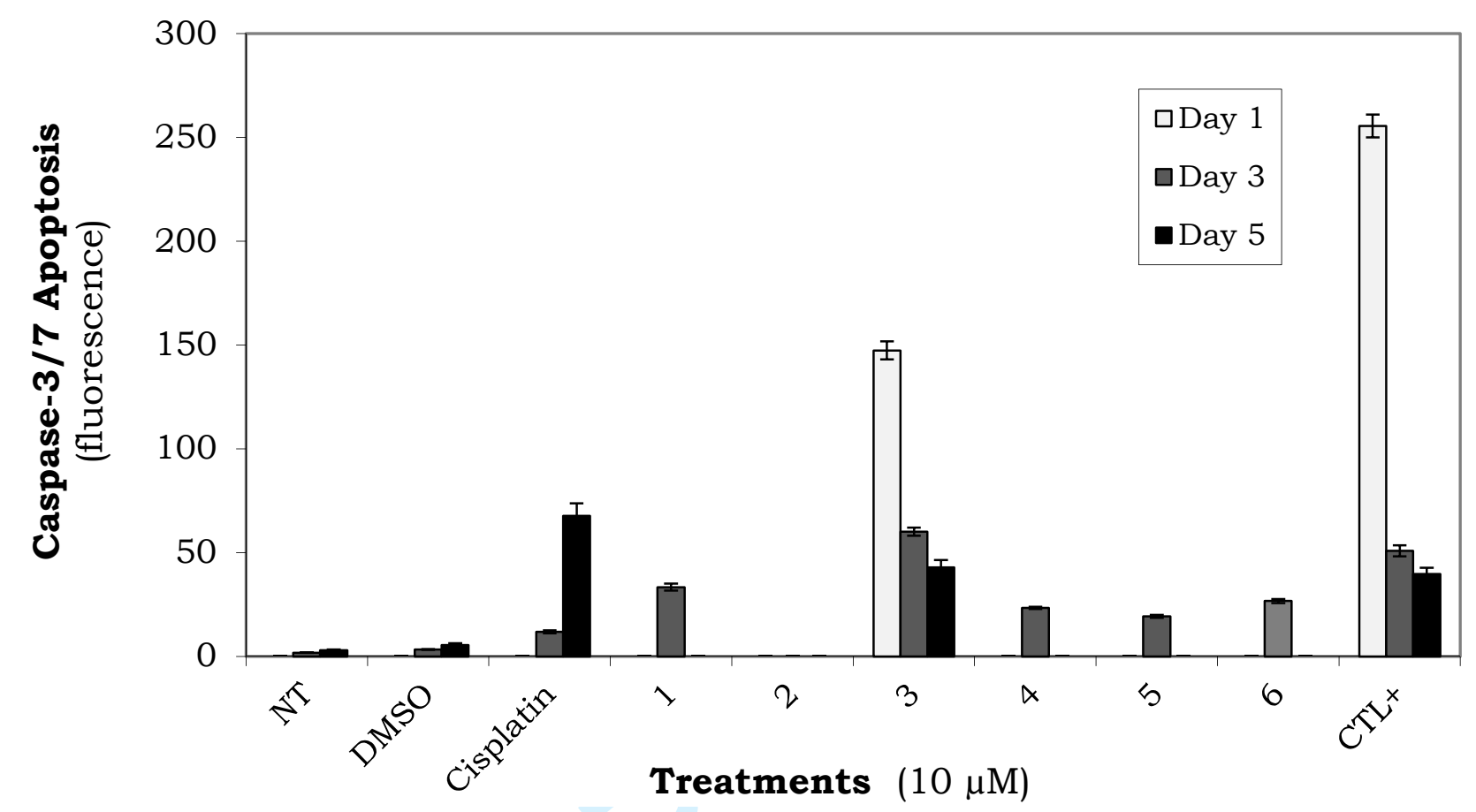

(b)

Figure 3. Platinum complexes $(10 \mu \mathrm{M})$ were incubated with MB231 breast cancer cells and evaluated for (a) cell viability and (b) caspase 3/7 activities over time (days 1,3 and 5) using a microscale cell-based assay. Control samples include non-treated (NT) parental cells; solvent (DMSO) treated cells; and, cells treated with apoptosis-inducing agent Melphalan $(5 \mu \mathrm{M})$ as a positive control $(\mathrm{CTL}+)$. Results and standard deviations are representative of biological and experimental triplicates.

To determine whether the observed growth arrest was due to apoptosis, a multiwell cellular assay based on the activities of effector caspases-3 and -7 was performed on MB231 breast cancer cells treated with the synthetized compounds. As expected, cisplatin induced caspase-dependent apoptosis in MB231 cells over 5 days of treatment in comparison to control samples (parental and solvent-treated) which did not display any apoptotic activity over time (Figure 3b). All new platinum complexes (except for 2) 
induced mild and brief apoptotic activity at $48 \mathrm{~h}$ post treatment. Compound $\mathbf{3}$ on the other hand, containing a long chain aliphatic group, induced a strong caspase-3/7 response within $24 \mathrm{~h}$ post-treatment which persisted for the remaining time course $(5$ days). Interestingly, compound 3 demonstrated a 147- and 5-fold increase in apoptosis at day 3 and day 5, respectively, when compared to the treatments from the commonly known cisplatin lead structure. Altogether, we observe that compound $\mathbf{3}$ is a more potent inducer of early apoptosis when compared to cisplatin in the malignant MB231 breast cancer cell line.

Compounds $\mathbf{1}-\mathbf{6}$ were then tested in Renal Cell Carcinoma (RCC) cell lines and compared to cisplatin. One genetic characteristic of RCC is related to the von HippelLindau (VHL) tumor suppressor gene that is inactivated by mutation and hypermethylation in up to $85 \%$ of cases. We chose three different RCC models with inactivated VHL (Figure 4) and their genetically matched counterpart with the functional VHL gene. ${ }^{35}$ In addition, the toxicities of these compounds was evaluated in a kidney epithelial normal cell line. As found in the literature, renal cancer cells were resistant to cisplatin in all models of carcinoma tested. Interestingly, the normal cells were also insensitive to cisplatin concentration tested. On the other hand, RCC were sensitive to compounds $\mathbf{1}$ and $\mathbf{2}$ prepared from long-chain amines, as observed in cells with $\mathrm{IC}_{50}$ between $7.10 \mu \mathrm{M}$ for compound $\mathbf{1}$ in RCC4/VHL and $17.45 \mu \mathrm{M}$ with compound 2 in RCC10 (Table 2). No significant difference was observed in response to compound $\mathbf{3}$ in renal cancer cells. Interestingly, compounds with diverse aromatic amines are more toxic for $\mathrm{RCC}$ with $\mathrm{IC}_{50}$ around $2.25 \mu \mathrm{M}$ in RCC4/VHL cells for compound $\mathbf{5}$ and $5.7 \mu \mathrm{M}$ in 786-0 cells in response to compound 4. Cytotoxicities were similar for compounds 4-6 and independent of VHL status. However, a high level of cytotoxicity was also 
observed in the normal kidney cells, suggesting that these compounds could induce potential nephrotoxicity in normal tissue. Further structural modifications and testing in normal and cancer cells could help to improve toxicity selectively for cancer cells.
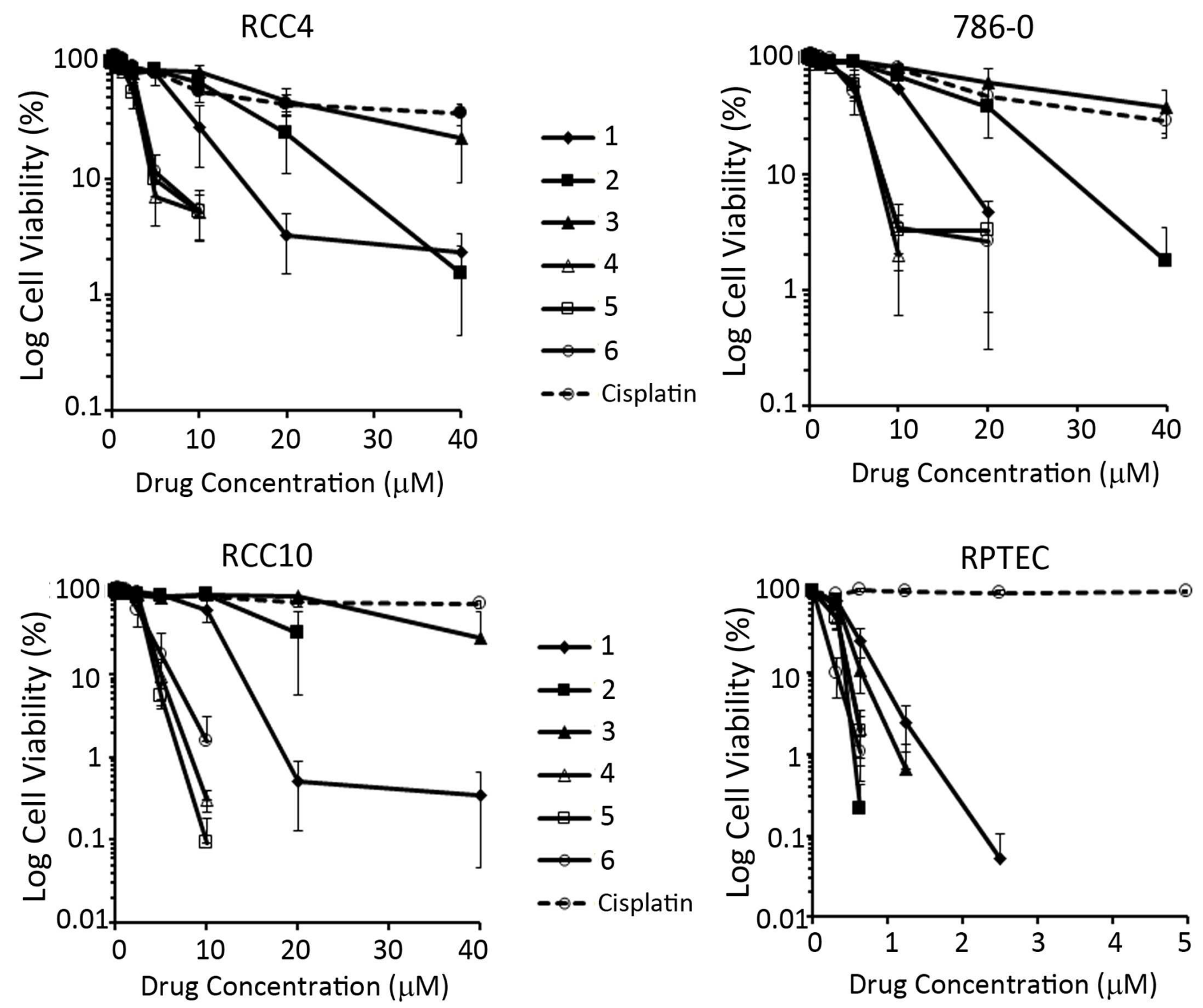

Figure 4. Effect of platinum compounds on renal cancer cells and normal renal proximal tubule cells. 
Table 2. $\mathrm{IC}_{50}$ for platinum complexes in different RCC models with, and without, VHL.

\begin{tabular}{|l|l|l|l|l|l|l|l|}
\hline & $\mathbf{1}$ & $\mathbf{2}$ & $\mathbf{3}$ & $\mathbf{4}$ & $\mathbf{5}$ & $\mathbf{6}$ & Cisplatin \\
\hline RCC4 & 7.496 & 13.65 & 21.68 & 2.838 & 2.520 & 3.085 & 13.69 \\
\hline RCC4/VHL & 7.105 & 12.34 & 22.87 & 2.411 & 2.242 & 2.637 & 7.066 \\
\hline RCC10 & 10.73 & 17.45 & 33.01 & 3.585 & 3.235 & 2.836 & $>40$ \\
\hline RCC10/VHL & 10.68 & 16.77 & 26.55 & 3.458 & 3.123 & 2.81 & $>40$ \\
\hline $786-0$ & 10.59 & 15.33 & 29.22 & 5.702 & 5.310 & 4.868 & 20.09 \\
\hline $786-0 /$ VHL & 9.923 & 16.12 & 30.64 & 5.572 & 5.262 & 4.527 & 20.71 \\
\hline RPTEC & 0.4421 & 0.337 & 0.385 & 0.3064 & 0.3033 & 0.1225 & $>40$ \\
\hline
\end{tabular}

\section{Conclusions}

In summary, we have prepared a small family of organometallic platinum complexes containing a chloride, cis-cyclooctene, and a Schiff base ligand. Fine-tuning the steric and electronic properties of the complexes was done by variation of the starting amine used to make the Schiff base ligands with salicylaldehyde. Three aliphatic amines with varying chain lengths and four aromatic amines with electron-donating and electronwithdrawing properties were chosen as representative examples. Although the complex containing an electron-withdrawing boron group (7) proved not to be stable in air, the remaining six complexes were examined for potential anticancer activities. Interestingly, complex 3, which contained the longest aliphatic chain studied (an octyl group), was the most promising for inducing apoptosis in the malignant MB231 breast cancer cell line. Conversely, complexes 4-6, which contained the aromatic groups, were the most active against renal cell carcinoma $(\mathrm{RCC})$ cell lines. Unfortunately, these complexes also showed significant cytotoxicity towards normal cell lines. Although these results are 
overall promising, further work is clearly needed to design a platinum complex based on this structural motif that is selective only towards cancerous cells.

\section{Supplemental material}

Crystallographic data for $\mathbf{4}$ and $\mathbf{7}$ have been deposited with the Cambridge Crystallographic Data Centre as supplementary publication numbers CCDC 1046057 and 1046058, respectively. Copies of the data can be obtained free of charge via www.ccdc.cam.ac.uk/conts/retrieving.html (or from the Cambridge Crystallographic Data Centre, 12 Union Road, Cambridge CB2 1EZ, UK; fax: + 441223336033 or e-mail: deposit@ccdc.cam.ac.uk).

\section{Acknowledgements}

We thank the Natural Science and Engineering Research Council of Canada, Mount Allison University, and the Université de Moncton for the financial support. AEP thanks the Beatrice Hunter Cancer Research Foundation for a summer grant. Dan Levon Durant is thanked for his expert technical assistance and anonymous reviewers thanked for their helpful comments. GAR is supported by grants from the Canadian Institutes of Health Research, the Canadian Breast Cancer Foundation-Atlantic Chapter, and the Canadian Breast Cancer Society/QEII Foundation. ST is recipient of a New Investigator award from the KRESCENT program and this work is supported by grants from the Cancer Research Society, New Brunswick Innovation Foundation and New Brunswick Health Research Foundation.

\section{References}

1. Wilson, J. J.; Lippard, S. J. Chem. Rev. 2014, 114, 4470-4495. 
2. Pinato, O.; Musetti, C.; Sissi, C. Metallomics 2014, 6, 380-395.

3. Pinato, O.; Musetti, C.; Farrell, N. P.; Sissi, C. J. Inorg. Biochem. 2013, 122, 27-37.

4. Bruijnincx, P. C. A.; Sadler, P. J. Curr. Opin. Chem. Biol. 2008, 12, 197-206.

5. $\quad$ van Rijt, S. H.; Sadler, P. J. Drug Discov. Today 2009, 14, 1089-1097.

6. Proetto, M.; Liu, W.; Hagenbach, A.; Albram, U.; Gust, R. Eur. J. Med. Chem. 2012, $53,168-175$.

7. Patterson, A. E.; Miller, J. J.; Miles, B. A.; Stewart, E. L.; Melanson, J.-M. E. J.; Vogels, C. M.; Cockshut, A. M.; Decken, A.; Morin Jr. P.; Westcott, S. A. Inorg. Chim. Acta 2014, 45, 88-94.

8. Klein, A.; Lüning, A.; Otto, I.; Hamel, L.; Neugebauer, M.; Butsch, K.; Lingen, V.; Heinrich, F.; Elmas, S. J. Organomet. Chem. 2010, 695, 1898-1905.

9. Alama, A.; Tasso, B.; Novelli, F.; Sparatore, F. Drug Discov. Today 2009, 14, 500508.

10. Enders, M.; Görling, B.; Braun, A. B.; Seltenreich, J. E.; Reichenbach, L. F.; Rissanen, K.; Nieger, M.; Luy, B.; Schepers, U.; Bräse, S. Organometallics 2014, 33, 4027-4034.

11. Shaver, M. P.; Vogels, C. M.; Wallbank, A. I.; Hennigar, T. L.; Biradha, K.; Zaworotko, M. J.; Westcott, S. A. Can. J. Chem. 2000, 78, 568-576.

12. Matsushita, T.; Shono, T. Polyhedron 1983, 2, 613-617.

13. Sheldrick, G. M. CELL_NOW, V. 2008/2, Bruker AXS Inc., Madison, Wisconsin, USA.

14. SAINT 7.23A, 2006, Bruker AXS, Inc., Madison, Wisconsin, USA.

15. Sheldrick, G. M. TWINABS 1.05, 2004, Bruker Nonius, Inc., Madison, Wisconsin, USA.

16. Sheldrick, G. M. Acta Cryst. A 2008, 64, 112-122. 
17. Sheldrick, G. M. SADABS 2008, Bruker AXS, Inc., Madison, Wisconsin, USA.

18. Komiya, N.; Kashiwabara, T.; Iwata, S.; Naota, T. J. Organomet. Chem. 2013, 738, 66-75.

19. Cui, J.; Zhang, M.; Zhang, Y. Inorg. Chem. Commun. 2010, 13, 81-85.

20. Zheng, F.; Hutton, A. T.; van Sittert, C. G. C. E.; Moss, J. R.; Mapolie, S. F. Dalton Trans. 2013, 42, 11163-11179.

21. Liu, S.; Sun, H.; Ma, Y.; Ye, S.; Liu, X.; Zhou, X.; Mou, X.; Wang, L.; Zhao, Q.; Huang, W. J. Mater. Chem. 2012, 22, 22167-22173.

22. Komiya, N.; Itami, N.; Naota, T. Chem. Eur. J. 2013, 19, 9497-9505.

23. Kaplan, S. F.; Kukushkin, V. Y.; Shova, S.; Suwinska, K.; Wagner, G.; Pombeiro, A. J. L. Eur. J. Inorg. Chem. 2001, 1031-1038.

24. Komiya, N.; Okada, M.; Fukumoto, K.; Kaneta, K.; Yoshida, A.; Naota, T. Chem. Eur. J. 2013, 19, 4798-4811.

25. Komiya, N.; Muraoka, T.; Iida, M.; Miyanaga, M.; Takahashi, K.; Naota, T. J. Am. Chem. Soc. 2011, 133, 16054-16061.

26. Komiya, N.; Okada, M.; Fukumoto, K.; Jomori, D.; Naota, T. J. Am. Chem. Soc. 2011, 133, 6493-6496.

27. Hawkeswood, S.; Stephan, D. W. Dalton Trans. 2005, 2182-2187.

28. Qu, Y.; Fitzgerald, J. A.; Rauter, H.; Farrell, N. Inorg. Chem. 2001, 40, 6324-6327.

29. Williams, J. W.; Qu, Y.; Bulluss, G. H.; Alvorado, E.; Farrell, N. P. Inorg. Chem. 2007, 46, 5820-5822.

30. Khiati, S.; Luvino, D.; Oumzil, K.; Chauffert, B.; Camplo, M.; Barthélémy, P. ACS Nano 2011, 5, 8649-8655.

31. Jansen, B. A. J.; Wielaard, P.; Kalayda, G. V.; Ferrari, M.; Molenaar, C.; Tanke, H. J.; Brouwer, J.; Reedijk, J. J. Biol. Inorg. Chem. 2004, 9, 403-413. 
32. Kalayda, G. V.; Jansen, B. A. V.; Molenaar, C.; Wielaard, P.; Tanke, H. J.; Reedijk, J. J. Biol. Inorg. Chem. 2004, 9, 414-422.

33. Kahlert, J.; Austin, C. J. D.; Kassiou, M.; Rendina, L. M. Aust. J. Chem. 2013, 66, 1118-1123.

34. Benzina, S.; Harquail, J.; Jean, S.; Beauregard, A.-P.; Colquhoun, C. D.; Carroll, M.; Bos, A.; Gray, C. A.; Robichaud, G. A. Anticancer Agents Med. Chem. 2015, 15, 79-88.

35. Chan, D. A.; Sutphin, P. D.; Nguyen, P.; Turcotte, S.; Lai, E. W.; Banh, A.; Reynolds, G. E.; Chi, J.-T.; Wu, J.; Solow-Cordero, D. E.; Bonnet, M.; Flanagan, J. U.; Bouley, D. M.; Graves, E. E.; Denny, W. A.; Hay, M. P.; Giaccia, A. J. Sci. Transl. Med. 2011, 3, 94ra70. 
Table 1. Crystallographic Data-Collection Parameters

\begin{tabular}{|c|c|c|}
\hline Complex & 4 & 7 \\
\hline Formula & $\mathrm{C}_{21} \mathrm{H}_{24} \mathrm{ClNOPt}$ & $\mathrm{C}_{27} \mathrm{H}_{35} \mathrm{BClNO}_{3} \mathrm{Pt}$ \\
\hline Molecular weight & 536.95 & 662.91 \\
\hline Crystal system & Monoclinic & Monoclinic \\
\hline Space group & $\mathrm{C} 2 / \mathrm{c}$ & $\mathrm{P} 2(1) / \mathrm{n}$ \\
\hline$a / \AA$ & $29.381(10)$ & $11.310(3)$ \\
\hline$b / \AA$ & $7.0580(19)$ & $20.960(5)$ \\
\hline$c / \AA$ & $20.989(5)$ & $11.570(3)$ \\
\hline$\alpha / o$ & 90 & 90 \\
\hline$\beta / \mathrm{o}$ & $117.996(17)$ & $97.588(4)$ \\
\hline$\gamma / \mathrm{o}$ & 90 & 90 \\
\hline$V / \AA^{3}$ & $3843.2(19)$ & $2718.8(12)$ \\
\hline$Z$ & 8 & 4 \\
\hline $\rho_{\text {calc. }} / \mathrm{Mg} \mathrm{m}^{-3}$ & 1.856 & 1.620 \\
\hline Crystal size $/ \mathrm{mm}^{3}$ & $0.30 \times 0.20 \times 0.12$ & $0.40 \times 0.15 \times 0.05$ \\
\hline Temp/K & $173(1)$ & $198(1)$ \\
\hline Radiation & Mo- $K_{\alpha}(\lambda=0.71073 \AA)$ & $\operatorname{Mo}-K_{\alpha}(\lambda=0.71073 \AA)$ \\
\hline$\mu / \mathrm{mm}^{-1}$ & 7.449 & 5.287 \\
\hline Total reflections & 6181 & 18805 \\
\hline $\begin{array}{l}\text { Total unique } \\
\text { reflections }\end{array}$ & 3844 & 6117 \\
\hline No. of variables & 244 & 324 \\
\hline$\theta$ Range/o & 1.57 to 27.49 & 1.94 to 27.50 \\
\hline $\begin{array}{l}\text { Largest difference } \\
\text { peak/hole/e } \AA^{-3}\end{array}$ & 3.487 and -1.828 & 1.203 and -0.758 \\
\hline $\mathrm{S}(\mathrm{GoF})$ on $\mathrm{F}^{2}$ & 1.099 & 1.028 \\
\hline$R 1^{\text {a }}(I>2 \sigma(I))$ & 0.0452 & 0.0274 \\
\hline $\mathrm{w} R 2^{\mathrm{b}}$ (all data) & 0.1419 & 0.0730 \\
\hline
\end{tabular}

a) $\left.R 1=\sum\left\|F_{\mathrm{o}}|-| F_{\mathrm{c}}\right\| / \sum\left|F_{\mathrm{o}}\right| .{ }^{b}\right) \mathrm{w} R 2=\left(\sum\left[\mathrm{w}\left(F_{\mathrm{o}}{ }^{2}-F_{\mathrm{c}}{ }^{2}\right)^{2}\right] / \sum\left[\mathrm{w} F_{\mathrm{o}}{ }^{4}\right]\right)^{1 / 2}$, where $\mathrm{w}=1 /\left[\sigma^{2}\left(F_{\mathrm{o}}{ }^{2}\right)+(0.0922 \cdot P)^{2}\right]$ (4), $1 /\left[\sigma^{2}\left(F_{\mathrm{o}}{ }^{2}\right)+(0.0325 \cdot P)^{2}+(3.1369 \cdot P)\right](\mathbf{7})$, where $P=\left(\max \left(F_{\mathrm{o}}{ }^{2}, 0\right)+2 \cdot F_{\mathrm{c}}{ }^{2}\right) / 3$. 